\title{
Zofia Berdychowska
}

\section{Personreferenz in linguistischen Abschlussarbeiten polnischer Germanistikstudierender im Vergleich mit dem deutschen linguistischen Usus}

\begin{abstract}
By comparing the forms of personal deixis in BA and MA theses written by Polish students with person reference in scientific writing by German linguists, this paper points out factors influencing their choice against the background of systemic differences between German and Polish as well as the functional dynamics of grammaticalized exponents of personal deixis.
\end{abstract}

Keywords: scientific writing, person reference, language comparison, German and Polish

\section{Problemstellung}

Studierende aller Studienrichtungen werden spätestens bei der Abfassung ihrer Abschlussarbeiten ${ }^{1}$ vor mehrere Probleme gestellt, die sie in Seminaren mit Hilfe von Betreuern und durch Fachlektüre zu lösen versuchen. Zu dem sog. wissenschaftlichen Schreiben ${ }^{2}$ liegen als Hilfesteller bereits zahlreiche artund sprachverschiedene Leitfaden und Ratgeber, darunter sowohl deutsche als auch polnische, für geisteswissenschaftliche und ebenfalls für andere Fächer vor, eine Sonderstellung nehmen spezielle monographische Betrachtungen von Metatextemen ein. ${ }^{3}$ In linguistischen Abschlussarbeiten sollten Studierende die Fähigkeit nachweisen, Sprachphänomene und den Prozess der Kommunikation tiefgründig zu analysieren und zu interpretieren, wie auch Schlussfolgerungen zu formulieren, die die Ergebnisse dieser Operationen zusammenfassen.

1 Gemeint sind hier grundsätzlich Lizenziats- und Magisterarbeiten, die textsortenmäßig zwar als wissenschaftliche Beiträge aufzufassen sind, dem Anspruch nach jedoch sich vielmehr als prä-wissenschaftlich einstufen lassen.

2 Vgl. Anm. 1. Der Bezeichnung „wissenschaftliches Schreiben“ für alle mit der Entwicklung der Textproduktionskompetenz im wissenschaftlichen Diskurs zusammenhängenden Aktivitäten ziehe ich deshalb die Bezeichnung „akademisches Schreiben“ vor, wie sie in der jüngst erschienenen Bibliographie von Cirko et al. (2017) genannt werden.

3 Exemplarisch seien hier erwähnt Gajewska (2004) und Olszewska (2007). 
Bewährte Faustregeln, die an Studierende in Seminaren vermittelt werden und anzuwenden sind, thematisieren u. a. die Erwartung, dass sich in der Arbeit auch das eigene Gedankengut der Verfasser niederschlägt. In Seminaren weisen die Dozenten darauf hin, dass durch die herangezogene Fachliteratur die Ebene eigener Bezüge und Begründungen für eigene Behauptungen und Bewertungen aufgebaut wird und dass bei der Bezugnahme auf Fachliteratur eigene Gedanken von Auffassungen Anderer deutlich abzugrenzen sind. Vorgegeben werden im Allgemeinen objektiver, klarer Stil, richtige Auswahl der Fachliteratur, sinnvolle und nachvollziehbare Gliederung der Arbeit, präzise Definition der Schlüsselbegriffe anhand der Analyse der in der Fachliteratur angewandten Definitionen, logische Darstellung der Argumente und Gegenargumente, klare Abgrenzung zwischen fremder und eigener Stellungnahme, Begründung der eigenen Auffassung, Verweise auf die dem Text zugrunde liegenden Quellen, Belegverweise für direkte und indirekte Zitate, Gebrauch der für den wissenschaftlichen Diskurs typischen Ausdrücke und der Terminologie. ${ }^{4}$ Wie nicht selten scheinen Interessen der Studierenden und der Dozenten etwas andersgeartet zu sein. Während Dozenten Nachdruck auf Zitierweise und auf den Umgang mit dem Gedankengut anderer Autoren durch bibliographische Verweise legen sowie mit Nachdruck vor Plagiaten warnen, bringen (nicht nur) Anfänger im akademischen (nicht nur fremdsprachlichen) Schreiben ihre Unsicherheit im Gebrauch personreferenzieller Formen zum Ausdruck. Diese Unsicherheit, teilweise sogar auch Irritation darüber, veranlasst zur Beschäftigung mit dem Vollzug der Personreferenz in akademischen Abschlussarbeiten.

\section{Vorgaben und Unsicherheit}

Unsicherheit im Personverweis bzw. -referenz kennzeichnet nicht nur angehende Germanisten, die an ihren Abschlussarbeiten schreiben. In Verwirrung geraten ebenfalls Studierende anderer geisteswissenschaftlicher Studienrichtungen wie auch Physiker und Informatiker, nicht selten auch Doktoranden. Ihre Frage nach dem Wie? bleibt entweder unangesprochen bzw. nicht genügend geklärt oder resultiert einerseits in eindeutig formulierten Geboten einer unpersönlichen Sprachverwendung, andererseits in Verboten, sich selbst als Textverfasser und eigene Meinungen durch ich-Bezug in den Text zu bringen mit der Begründung, dass Konventionen des wissenschaftlichen Schreibens nicht den Verfasser,

4 http://www.ifg.filg.uj.edu.pl/studenci/materialy [16.02.2017]. 
sondern die Sache selbst, das erforschte Phänomen in den Vordergrund treten und objektiv darstellen lassen. ${ }^{5}$

Repräsentativ für die um die Formen der auktorialen Referenz gestifteten Verwirrung ist die Diskussion auf einem Doktorandenforum, die hier stichwortartig wiedergegeben sei: Die Frage nach korrekten sprachlichen Formen der auktorialen Referenz in wissenschaftlichen Arbeiten - Ausdruck der Unsicherheit in Reaktion auf wiederholte Korrekturen - stellt ein Promovierter, in dessen Dissertation und wissenschaftlichen Beiträgen sowohl die 1. Person Singular vom Doktorvater als auch die Form der 1. Person Plural von Mitverfassern weiterer Beiträge zu polnischen unpersönlichen präteritalen -no/-to-Formen stets korrigiert wurden und werden, während die Handhabung der auktorialen Referenz im fremdsprachlichen Schrifttum lockerer sei. Die Reaktionen auf dem Forum bewegen sich zwischen Ablehnung und Befürwortung der Korrekturen und weisen wiederholt die Unsicherheit im Umgang mit Formen der auktorialen Referenz auf. Argumente für die Verwendung unpersönlicher Formen gehen eindeutig auf die Vorgaben und Argumente der Betreuer zurück: Die kanonische Form der Personreferenz mit dem Pronomen der 1. Person Singular sei zu keck, angeberisch, denn dadurch werde die Aufmerksamkeit auf den Verfasser und nicht auf das Referierte gelenkt und was sie [die angehenden Wissenschaftler] schreiben, sei doch keine offenbarte Wahrheit; durch anonymisierende Formen werde der Leser informiert, dass der Verfasser Distanz zum Erforschten und Beschriebenen bewahrt. Andere dagegen bringen - mit Recht - die Überzeugung und auch die Bewusstheit über die verantwortungsmäßige Äußerungsträgerschaft zum Ausdruck, wenn der Äußerungsträger zugleich die Quelle der Mitteilung ist und sich als solcher identifiziert, etwa nach dem Motto: Ich schreibe darüber, was ich gemacht und ermittelt habe und was ich denke. Dabei interpretieren die Diskutanten die Wirkung aller infrage kommenden Formen der auktorialen Personreferenz uneinheitlich und wertend. Am stärksten wenden sie sich gegen die wir-Form des Vollzugs einer singulären auktorialen Referenz. ${ }^{6}$ Ihre ablehnende Haltung der mit dem Pronomen wir unklar ausgeführten Referenz gegenüber folgt nach Graefen (1997: 214) aus dem Widerspruch zwischen dem sprachlichen Phänomen und der Realität, für dessen Bewältigung propositionale und illokutive Merkmale der Äußerung herangezogen werden

5 Im Gegensatz zur Werbebranche, die - obwohl auch nicht auf Personen, sondern Produkte fokussiert - durch den Gebrauch der 1. P. Sg. in den vermeintlichen Kundenaussagen eine Identifizierung des Rezipienten mit der Aussage anstrebt.

6 http://forum.(gazeta.pl/forum/w,87574,111206136,111206136,Strona_bezosobowa_w_artykulach_i_tak_dalej_html [16.02.2017]. 
müssen. ${ }^{7}$ Die Bewusstheit und eigene Überzeugung von der Richtigkeit des ebenfalls in deutschen wissenschaftlichen Texten von manchen Linguisten tabuisierten $i c h$-Verweises ${ }^{8}$ werden zusätzlich durch die im Netz aktiven Dozenten und Korrekturfirmen erschüttert. ${ }^{9}$ So bleibt bei jungen Produzenten akademischer Texte die Unsicherheit über die zu befolgende „Norm“ bestehen, wobei Norm und Konvention und demzufolge Korrektheit, Akzeptanz und Angemessenheit vermengt werden.

\section{$3 \mathrm{Zu}$ analyserelevanten deutschen und polnischen Formen der Personreferenz in wissenschaftlichen Texten}

Die Personreferenz ist im Rahmen und auf dem Hintergrund des gesamten Sprachsystems und des Sprachgebrauchs in konkreten Kommunikationsereignissen zu betrachten. Wie alle Sprachmittel werden auch die auf Person bezogenen immer in einer Relation zum Satz, zur Äußerung, zum Text und zur gesamten Kommunikationssituation, in denen sie verwendet werden, wie auch in Relation zu dem im Langzeitgedächtnis gespeicherten und das Laufwissen anreichenden Dauerwissen des Textproduzenten selbst, dem beim Textrezipienten vorausgesetzten und seinem tatsächlich verfügbaren bzw. dem beiden Kommunikationspartnern gemeinsamen Wissen semantisch und/oder konzeptuell verarbeitet und interpretiert. ${ }^{10}$ Denn es gehört zur Spezifik personreferenzieller Mittel, dass sie mehr oder weniger genau angeben können, auf wen sie verweisen. Oft signalisieren sie lediglich, dass ein Verweis angelegt wurde: „Man kann nicht einmal eindeutig erkennen, welcher Art der Verweisraum sein könnte. Erst im kommunikativen Gebrauch werden Konventionen wirksam, die eine Bestimmung

7 Auszählungen von vor zwanzig Jahren von Graefen (1997: 208) weisen die wir-Form als dominant in wissenschaftlichen Texten aus. In der Polonistik ist die auktoriale wirReferenz nicht allgemein üblich, kommt aber noch vor.

8 Vgl. Literaturangaben dazu in Szurawitzki (2011: 79).

9 Vgl. u. a. https://www.ii.pwr.edu.pl/ kazienko/mgrwytyczne.pdf; http://www.pisanie-prac.info.pl/prace-magi-sterskie-jak-pisac.php; http://wiedza-naukowa.eu/stylnaukowy-czym-sie-charakteryzuje/; http://jerzy_woyke.users.sggw.pl/jakniepisac.html [(16.02.2017)].

10 Welche von diesen Elementen des Wissens und des Kontextes i. w. S. bei der Produktion und Interpretation einer Äußerung relevant sind, ist nicht voraussehbar (Levinson 1990: 23-24). Zu auf Schwarz (2000) aufbauenden lexikon- und weltwissensbasierten Modellen der Verarbeitung der Personreferenz vgl. Berdychowska 2002, Kap. 6.2. Liedtke (1998: 265-273) weist auf die Satztyp/Illokutionstyp-Korrelation als Interpretationsgrundlage in seinem Modell der Kontexthierarchien hin. 
des Verweisraums und gegebenenfalls des Gegenstands oder des Gegenstandsentwurfs unterstützen." (Zifonun et al. 1997: 747-748). Wenn senderseits die Verbergung der kognitiven Adresse der Bezugnahme auf Person intendiert ist, wählt er auf Nicht-Identifikativität spezialisierte Mittel, die unter bestimmten ko- und kontextuellen Umständen und/oder Sprechhandlungsbedingungen auf Personen spezifisch bezogen werden können. Da im Vollzug der Personreferenz in Abschlussarbeiten polnischer Germanistikstudierender der Einfluss ihrer L1 nicht auszuschließen ist, seien die relevanten Formen im Deutschen und im Polnischen synoptisch ins Auge gefasst.

Dem Polnischen und dem Deutschen ist das sechsstellige System der Personalpronomen gemeinsam. ${ }^{11}$ Sie können auf Personen in der Sender- oder Adressatenrolle grundsätzlich identifizierend und in der Rolle des Besprochenen reidentifizierend bezogen werden. Nur unter bestimmten Voraussetzungen werden sie generisch verwendet. Bedeutende Unterschiede weist der Formenbestand der auf Nicht-Identifikativität spezialisierten Mittel auf. Im Polnischen findet sich im pronominalen System keine Entsprechung für die extreme Breite des Extensionspotentials des deutschen Pronomens man. Das Polnische verfügt dagegen $u$. a. über nichtidentifikative anonymisierende genusneutrale Konstruktionen mit präteritalen prädikativen Formen auf -no, - $-\mathrm{o}^{12}$ (Wybito okno. ,das Fenster wurde eingeschlagen') mit inkludierten Subjekten, die nicht freigelegt, d. h. nicht explizit genannt werden können und rein kontextuell bedingte, durch die Verbsemantik unterstützte Referenz haben. Ebenfalls rein kontextuell bedingte Referenz haben Konstruktionen mit Modalprädikativen (Szkoda czasu. ,schade um die Zeit') und Konstruktionen aus einem Modalprädikativ mit dem Infinitiv (z. B.: można - Möglichkeit, Erlaubnis: Tezę pracy można sformułować następująco. ,die These der Arbeit kann folgendermaßen formuliert werden'). $\mathrm{Zu}$ nicht identifikativen Formen gehören des Weiteren die 3. Person Plural von Verben mit strukturell blockiertem Subjekt (Wybili okno. ,das Fenster wurde eingeschlagen') und impersonale Konstruktionen mit dem Verbum in der 3. P. Sg. mit dem Pronomen sie, das nur die Nominativform besitzt, in die Position des Subjekts tritt und im Hinblick auf die Kategorie Person wie das deutsche man klassifizierend (d. h. ausschließlich auf die Klasse Humanum bezogen) aufzufassen ist (Używa się obu form. ,man gebraucht beide Formen'/,beide Formen

11 Unterschiede, die für die im Fokus stehende Personreferenz in wissenschaftlichen Texten nicht relevant sind (u. a. Distanzformen, Referenzumfang der komitativ gebrauchten Deiktika $m y /$ wir, minimale Erweiterungsmöglichkeiten der Pronominalphrasen u. a. - vgl. Berdychowska 2013, Kap. 3.1), bleiben hier unbeachtet.

12 Auch die Form mit - Ło, die aber für akademisches Schreiben nicht relevant ist. 
werden gebraucht $\left.{ }^{\prime}\right)$. Ihr Gebrauch ist immer mit dem Effekt der Anonymisierung oder Typisierung verbunden, ähnlich wie es bei man der Fall ist. ${ }^{13}$ An den erläuternden deutschen man- und Passivparaphrasen für die oben nur unvollständig dargestellten nicht identifikativen Mittel im Polnischen wird deutlich, dass in ihrer Vielfalt nicht nur der seltenere Passivgebrauch im Polnischen als im Deutschen, sondern auch (und vor allem) die starke, möglicherweise durch Äquivalenzsuche bewirkte, darunter häufig unangemessene Repräsentation von man in Abschlussarbeiten begründet ist.

\section{Die Praxis}

Für eine hier nur exemplarische Analyse des Umgangs mit der Personreferenz in Abschlussarbeiten ${ }^{14}$ polnischer Germanistikstudierender habe ich je drei Lizenziats- und Masterarbeiten aus dem umfangreichen Korpus ${ }^{15}$ durch Zufallsauswahl bestimmt. Belege für die Verwendung der Formen ich, wir, man und Passiv werden in ihren originalen Formen angeführt, aber um Elemente gekürzt, die für die Analyse irrelevant sind.

Eine stereotype Einleitung am absoluten Textanfang enthält eine explizite Referenz auf den Autor und beginnt eher mit in meiner Arbeit als mit ich, z. B.: In meiner Arbeit möchte ich mich mit der sprachlichen Analyse solcher Texte befassen, die [...] (MA2004MT). Die gleiche, jedoch implikatierte Referenz wie die explizite auktorial referierende Phrase in meiner Arbeit/meine Arbeit hat die Phrase in der vorliegenden Arbeit/die vorliegende Arbeit, weil die vorliegende Arbeit die des Verfassers ist. Vgl. (1):

(1) Die vorliegende Arbeit setzt sich zum Ziel [...] zu beleuchten und zu zeigen, wie man mit $[\ldots]$ Informationen über [...] im Unterricht vermitteln kann. [...]

In dem ersten Kapitel wird [...] dargestellt. Es wird auch festgestellt, dass [...]. Die Unterkapitel handeln von [...]. Schließlich werden diese Ansätze gegenübergestellt und verglichen.

Anhand von [...] wird [...] dargestellt. [...] Es werden verschiedene Arbeitsformen für den Unterricht angeboten, die [...] den Lernenden zum Lernen motivieren werden. [...], wird in dem dritten Kapitel [...] beschrieben. (BA2007MW)

13 Eine vergleichende Gesamtdarstellung findet sich u. a. in Berdychowska (2002, Kap. 4).

14 Lizenziatsarbeiten $=\mathrm{BA}$; Masterarbeiten $=$ MA.

15 Das umfangreiche Material (über zweihundert Abschlussarbeiten von Germanistikstudenten aus den Jahren 2006-2016 in ihren Entstehungsphasen bis zur Endversion) konnte für eine exemplarische Betrachtung in dem Beitrag nur punktuell ausgewertet werden. Keine Rolle spielten bei der Auswahl die Leistungen der Verfasser - sie bewegen sich zwischen sehr schwach und ausgezeichnet. 
Die Nominalphrasen meine Arbeit/die vorliegende Arbeit sind stereotype Versuche, die ich-Form (nicht aber die auktoriale Referenz) am Textbeginn zu meiden. Die ebenso stereotype anthropomorphisierende Fortsetzung des Satzes mit setzt sich zum Ziel versetzt den Autor noch stärker in den Hintergrund.

(2) In diesem Kapitel wird [...] dargestellt, wie [...] präsentiert werden kann. [...]

Ich habe dieses Land gewählt, weil ich dort ein Jahr gewohnt habe. Außerdem finde ich das Land sehr interessant und spannend, [...]. [...] denn ich denke, [...]. (BA2007MW)

(3) Gegenstand dieses Kapitels ist [...]. Bei der Behandlung dieser Frage möchte ich mich nur auf die wichtigsten Aspekte beschränken. Was versteht man unter den Begriffen Wörterbuch, Glossar und Thesaurus? Wie ist ihre Struktur? Zu welchem Zweck werden sie benutzt? Diesen Fragen wird im Folgenden besonderes Augenmerk geschenkt. (BA2015KN)

Auch ein neues Kapitel einleitende Passagen enthalten den ich-Bezug erst in einem zweiten Satz, in dem u. a. Begründungen für eine Entscheidung oder Schwerpunkte des Kapitels genannt oder Forschungsfragen formuliert werden. Vgl. (2), (3).

(4) Anhand dieser Annahme stelle ich die Hypothese auf, dass [...]. Diese Annahme erlaubt auch, [...]. Darüber hinaus will ich [...] beschreiben, um [...] einzuordnen. Diesen Einordnungsbereich stellt [...] dar, die als [...] beschrieben wird ${ }_{\text {auktorial }}$ der [...]. XX wird ${ }_{\text {auktorial }}$ als [...] angesehen. Sie wird ${ }_{\text {auktorial }}$ als [...] verstanden. Schließlich soll diese theoretische Ausführung dazu dienen, zu zeigen, dass [...] Ein weiteres Feinziel der Arbeit ist es zu zeigen, dass [...] (MA2009GK)

Belege aus einleitenden Passagen zeigen, dass am Textanfang durch identifizierende Mittel Basis für die Verarbeitung und Interpretation der Personreferenz geschaffen wird. Der weitere Text befindet sich im Skopus der im einleitenden Satz mit der expliziten identifizierenden Personreferenz festgelegten Betrachtungsperspektive, die den Text organisiert und somit kohärenzstiftend wirkt. Daher kann die Passage durch Passivkonstruktionen ohne Agensanschluss fortgesetzt werden. Nicht identifikative Referenzmittel in darauffolgenden Sätzen werden als auf dieselbe Person wie im einleitenden Satz bezogen interpretiert, soweit kein Signal des Perspektivenwechsels oder einer Perspektivenunterbrechung durch eine neue identifizierende Personreferenz oder Sprachhandlungswechsel feststellbar ist. Besonders anschauliche Belege dafür sind (1) und (4).

(5) Widmen wir uns zuerst der Frage, wie [...] in der Forschung verstanden wird und welche Art der grammatischen Formen man unter dem Begriff [...] versteht. Im Folgenden wird $_{\text {auktorial }}[\ldots]$ als [...] verstanden. (MA2009GK)

Eine vergleichbare perspektivierende Funktion erfüllen die in den herangezogenen Arbeiten ganz seltenen wir-Vorkommen, sie sind zugleich an den potentiellen Rezipienten adressiert als Appell. Vgl (5). 
(6) Bei der Definition dieses Begriffs möchte ich mich auf die Worte von [...] stützen, die eine wichtige Basis des vorliegenden Kapitels darstellen. (BA2007MW)

(7) Ich finde, dass es vielleicht sinnvoller wäre, wenn man größeren Wert auf die sprachlichen Übungen gelegt hätte. (BA2002KI)

Ich-Bezüge im Haupttext erscheinen bei Ankündigungen weiterer Schritte und Anknüpfungen an bereits Gesagtes und dienen der Textorganisation (6). Die wertende auktoriale ich-Aussage (7), bzw. eine ganze Reihe gleich beginnenden Sätze mitten im Analyseteil des Haupttextes ist in der Endversion der Arbeit in den Schlussteil verlegt worden.

(8) In meiner Diplomarbeit wollte ich veranschaulichen und analysieren, [...]. Im Besonderen möchte ich auf $[\ldots]$ eingehen. (BA2002KI)

(9) Diese Aussage kann, meines Erachtens, nur dann Gültigkeit beanspruchen, wenn man [...]. (MA2009GK)

Im Schlussteil wird die definite auktoriale Referenz in Zusammenfassungen (8) und Schlussfolgerungen (9) hauptsächlich zur Verdeutlichung der Stellungnahme verwendet.

Mit einer Passivkonstruktion ist über den propositionalen Gehalt ein anonymisierender Referenzbezug auf den Textproduzenten, den adressierten Rezipienten oder eine generische Bezugnahme möglich. Eine Passivkonstruktion unmittelbar nach (ggf. zwischen) agenszugewandten Aktiv-Sätzen ergibt eine implizierte Identifizierung des personalen Bezugsobjekts wie in (1), (4), (5).

In den Arbeiten kommen auch andere passivische Formen der Unterdrückung der auktorialen Referenz vor, wie z. B. in (10):

(10) Durch die Präfigierung erfolgt die Modifikation der Semantik des Ausgangsverbs. (MA2009GK)

Eine andere Strategie besteht in der Anthropomorphisierung nichtpersonaler Objekte, wie in (11), (vgl. auch (1)):

(11) Die kognitive Sprachwissenschaft setzt sich [...] entgegen. (MA2004MT)

Zum Anderen wird durch unangebracht gebrauchte Existenzialsätze mit es gibt die Allgemeingültigkeit des Sachverhaltes bewirkt:

(12) Es gibt drei Typen von Neuansätzen: [...]. (MA2004MT)

Der Beleg (12) suggeriert, dass darin, dass drei Typen von Neuansätzen existieren, allgemeine Übereinstimmung erreicht ist, während aus der Fortsetzung des Textes ganz am Ende klar hervorgeht, dass es sich um die Unterscheidung von Typen nach einem konkreten Autor handelt. 
Die im Teil 3 angesprochene Breite des Extensionspotentials des Pronomens man wird in den Arbeiten voll ausgenutzt. Die Substitution durch jemand zeigt, dass man-Vorkommen als partikulär auf die interessierte Fachgemeinschaft bezogen interpretierbar sind, dagegen die Substitution durch jeder als generalisierend:

(13) Als [...] Lösung dieses Problems kann man [...]. (MA2006PD)

(14) In der [...] Forschung spricht man nämlich meistens von zwei Stellungnahmen [...].

(MA2009GK)

(15) Dabei stellt man fest, [...] (MA2006PD)

(16) [...] wobei man einen jeweilig untersuchten Text [...] mehreren Klassen zuordnet.

(MA2006PD)

(17) Nach Burger zählt man zu [...] (MA2006PD)

(18) Zusammenfassend kann man sagen, dass [...] (BA2007MW)

(19) Im Hinblick auf [...] kann man sehen, dass [...] (BA2007MW)

Über die diskursiv personreferenzielle Leistung von man sind die Meinungen geteilt. Während Panther (1981) dem Gebrauch von man die gleiche Möglichkeit der Bezugnahme auf den Sprecher als Mitglied einer (u. U. imaginären) Gruppe beimisst wie dem Gebrauch von wir, bestreitet Graefen (1997: 212), dass man die diskursive Qualität habe, erkennt jedoch dessen Potential, eine bereits auch mit Hilfe von anderen Deiktika mental für Interaktanten etablierte Gruppenzuordnung als Verstehensgrundlage aufrechtzuerhalten. Aufgrund des propositionalen Gehaltes ist man in (13) als auktoriale Referenz interpretierbar, durch Erwähnung der Wissenschaftsgemeinschaft in (14) als partikulärer Bezug. Eine unklare Personreferenz wie in (15) mindert nicht nur den Erkenntniswert derartiger Textpassagen, weil die Quelle einzelner Aussagen nicht angezeigt ist, sondern wirkt sich auch auf die Textorganisation und auf eine schwache Stiftung der Textkohärenz aus.

Wird das dynamische Extensionspotential von man nicht erkannt, erweist sich der Gebrauch von man als falsch, wenn zugleich eine konkrete Quelle angegeben wird, wie in (17). Da nichtidentifikative Ausdrücke dann zur Verwendung kommen, wenn der Sender auf eine Person referiert, die er nicht identifizieren kann oder will, steht das anonymisierend-generische man im Widerspruch zum gleichzeitigen identifizierenden Verweis mit dem Familiennamen. Die mögliche Lesart „Burger informiert über eine allgemein angenommene Systematisierung/ Einordnung" widerspricht auch der von der Verfasserin intendierten Darstellung der von Burger vorgenommenen/vorgeschlagenen Systematisierung/Einordnung. Auch (16), eine Formulierung, mit der die eigene Analyseprozedur geschildert wird, ist für die Erkennung der Personreferenz ungeeignet, weil verwirrend. Die referenziell unklare man kann-Routine, hinter der sich der 
Verfasser als Autor der zusammenfassenden Worte versteckt, ist in zusammenfassenden Textpassagen zu finden (18), (19).

Zum Schluss sei noch eine extreme Strategie erwähnt: In einer Arbeit findet sich kein ich, kein wir, sondern auf eine dritte Person referierende Aktivsätze beim Besprechen der Auffassungen, sonst Passivkonstruktionen und auktorial (nicht immer korrekt) intendierte man-Vorkommen. Eine Abgrenzung des fremden Textes erfolgt durch bibliographische Verweise im Haupttext und konventionelle Markierung der Zitate, welche wiederum beinahe durchgehend (sic!) man-Bezüge enthalten.

\section{Schlussfolgerungen}

Im Vergleich zu den Anforderungen erweist sich die Textproduktionskompetenz insbesondere all derjenigen, die - wie die polnischen Germanistikstudierenden - ihre Abschlussarbeiten in der Fremdsprache zu verfassen haben, zuweilen nicht in jeder Hinsicht als hinreichend. Die polnischen Germanistikstudierenden verfügen über hauptsächlich in den Grammatikvorlesungen und -seminaren erworbenes Wissen über den Formenbestand und Funktionen der Formen der Referenz auf Personen in ihren kommunikativen Rollen, jedoch weniger über Schreib- und Textroutinen, deren Beherrschung für eine erfolgreiche Wissenschaftskommunikation grundlegend, in der Praxis jedoch alles andere als einfach ist, weil Sprachgebrauch unter Einfluss von mehreren, teilweise schwer fassbaren Faktoren steht. So ist jungen VerfasserInnen akademischer Arbeiten bewusst, dass es einen Bedeutungsunterschied gibt zwischen: ich bin der Meinung, wir sind der Meinung, es wird die Meinung vertreten, man vertritt die Meinung u. ä., nicht aber im gleichen Maße auch, dass die Wahl der Formen zum Vollzug der auktorialen Referenz nicht (nur) durch die Grammatik geregelt ist, sondern von mehreren Faktoren, darunter von Textbauprinzipien und Textfunktionen abhängt und Konventionen unterliegt, die vielmehr vom wissenschaftlichen Diskurs bzw. von der Diskurskultur der jeweiligen Wissens- und Wissenschaftsgemeinschaft als von der Sprachgemeinschaft gesteuert werden. Unter Druck zum Gebrauch von Formen gesetzt, die das Agens anonymisieren oder den Senderbezug aus Gründen der Bescheidenheit unterdrücken, bringen sie vielleicht nicht so sehr ihre Irritation darüber wie eine Unsicherheit im Vollzug des auktorialen Bezugs zum Ausdruck.

Aus der nur überblicks- und stichprobeartigen Recherche in einem umfangreichen Material geht hervor, dass alle im akademischen Schreiben konventionalisierten Formen der Personreferenz in ihren jeweils zu realisierenden Funktionen - identifizierend, anonymisierend, typisierend, generalisierend und 
partikulär - verwendet werden, wenn auch nicht immer in erwünschter Weise mit der beabsichtigten oder zu erwartenden Wirkung. Wegen einer starken textuellen und kontextuellen Einbettung der mit Hilfe von nicht identifikativen Formen vollzogenen oder - wenn Formen mit einem nicht spezifizierten Subjekt oder Objekt gebraucht werden (vgl. oben Teil 3) - nur angelegten Personreferenz würden Versuche von vergleichenden Auszählungen der verwendeten Mittel für die Interpretation des Personbezuges keine schlüssigen Ergebnisse bereitstellen. Ihre nur beobachtungsmäßig unterschiedliche Gebrauchshäufigkeit weist zweifellos das extrem extensional flexible man, insbesondere in einer in den Abschlussarbeiten offensichtlich routinisierten Verbindung mit kann, aber auch mit anderen (Modal-)Verben, als dominante Form und gleichzeitig sprachliche Strategie aus, die Referenz sowohl auf sich selbst als Autor als auch auf Dritte mit anonymisierender Wirkung zu vollziehen, bzw. die scientific community inkludierend die partikuläre Geltung des Sachverhalts zu unterstellen. Verwischt wird dadurch zugleich die Grenze zwischen eigenen Gedanken und dem entlehnten Gedankengut, zwischen Referieren und Stellungnahme. Die Überbeanspruchung des Pronomens man und besonders der „man kann-Routine“ führt vielerorts zu den von Textproduzenten möglicherweise vielmehr verkannten als beabsichtigten Unklarheiten des Bezugs. Man kann ist insbesondere in leistungsschwachen Abschlussarbeiten zu einer routinisierten Allerwelts-Form geworden, die durch die Modalität auch dem Hedging dient. Etwas überraschend korreliert auch der massive Gebrauch von ich (ich finde ...), mit dem alle anderen Gesichtspunkte dominiert werden, mit schwacher Leistung. Ein ganz anderes Bild ergibt sich aus der Praxis des Vollzugs der Personreferenz in sehr guten Abschlussarbeiten. Die Autoren gebrauchen zweckmäßig vielfältige, auch der Hervorhebung der Erkenntnisse und ihrer Diskussion dienliche Mittel. Ihre Anordnung trägt zur Textorganisation wie zum Aufbau der Textkohärenz bei und ihre Wahl erlaubt eine deutliche Abgrenzung fremder und eigener Gedankengänge.

Die aufgespürte Routinisierung und Konventionalisierung der Vollzugsformen der Personreferenz wie auch mitunter ihr fehlerhafter Vollzug weisen auf einen nicht immer erkannten Zusammenhang mit Funktionen von Teiltexten und mit dem Vollzug der in einzelnen Textteilen dominanten Sprachhandlungen hin. Nicht unbegründet dürfte deshalb behauptet werden, dass die Handhabung historisch gewachsener Schreib- und Textroutinen in der wissenschaftlichen Kommunikation mit der Belesenheit in der Fachdisziplin stark positiv korreliert, die wiederum durch Erkenntnislust motiviert ist. Mit der Auswertung der Fachliteratur erwirbt man bestimmte Textbildungsmuster, darunter auch Muster für den Vollzug der Personreferenz, dem ich aufgrund der Analyse einen qualitätsindizierenden Wert zuschreibe. Da sich die in den 
Vorgaben angedeuteten funktionalen Stilprinzipien der wissenschaftlichen Kommunikation wie Unpersönlichkeit, Abstraktheit, Neutralität, Sachlichkeit, Folgerichtigkeit, Klarheit, Genauigkeit, Ökonomie aus der dominanten Funktion der wissenschaftlichen Texte ableiten, wäre eine deutlich stärkere Hervorhebung der Erkenntnis als Hauptfunktion akademischer Textproduktion sowohl dem angemessenen und klaren Vollzug der Personreferenz als auch einer kontroversen- bzw. persuasionsorientierten Konzipierung und Formulierung der Texte förderlich.

\section{Quellen}

Lizenziats- und Masterarbeiten von Germanistikstudierenden, angefertigt zwischen 2002-2016 (BA - an der Staatlichen Fachhochschule in Tarnów; MA an der Jagiellonen-Universität, Kraków).

\section{Literatur}

Berdychowska, Zofia (2002): Personaldeixis. Typologie, Interpretation und Exponenten im Deutschen und im Polnischen. Kraków: Universitas.

Berdychowska, Zofia (2013): Personendeixis. Kontraste Deutsch-Polnisch und ihre translatorischen Aspekte. Kraków: Wydawnictwo Uniwersytetu Jagiellońskiego.

Cirko, Lesław/Gołębiowski, Adam/Schönherr, Monika/Zuchewicz, Tadeusz (2017): Deutsche Ratgeber für das Fach „Akademisches Schreiben“: Ein bibliographischer Abriss 2000-2016. Wrocław: Atut; Dresden: Neisse Verlag.

Gajewska, Urszula (2004): Metatekstemy w języku nauk ścisłych. Rzeszów: Wydawnictwo Uniwersytetu Rzeszowskiego.

Graefen, Gabriele (1997): Der Wissenschaftliche Artikel - Textart und Textorganisation. Frankfurt/M.: Lang.

Levinson, Stephen C. (1990): Pragmatik. (Pragmatics, Cambridge University Press. (1983) Dt. Übers. von Ursula Fries. Tübingen: Niemeyer.

Liedtke, Frank (1998): Grammatik der Illokution. Über Sprechhandlungen und ihre Realisierungsformen im Deutschen. Tübingen: Narr.

Olszewska, Danuta (2007): Metatexteme in den Geisteswissenschaften. Typologie - Funktionalität - Stilistik. Gdańsk: Wydawnictwo Uniwersytetu Gdańskiego.

Schwarz, Monika (2000): Indirekte Anaphern in Texten. Tübingen: Niemeyer.

Szurawitzki, Michael (2011): Linguistische Untersuchungen zur strukturellen Gliederung, zur Verwendung von Personaldeixis und evaluativer Sprache in 
Rezensionen aus der Zeitschrift für deutsches Altertum und deutsche Literatur. In: Moderna språk 2011/1, 74-100.

Zifonun, Gisela/Hoffmann, Ludger/Strecker, Bruno (1997): Grammatik der deutschen Sprache. Berlin/New York: de Gruyter.

\section{Internetquellen}

http:/forum.gazeta.pl/forum/w,87574,111206136,111206136,Strona_bezosobowa_w_artyku-lach_i_tak_dalej_.html [16.02.2017] http://jerzy_woyke.users.sggw.pl/jakniepisac.html [16.02.2017] http://wiedza-naukowa.eu/styl-naukowy-czym-sie-charakteryzuje/ [16.02.2017]

http://www.ifg.filg.uj.edu.pl/studenci/materialy [16.02.2017] https://www.ii.pwr.edu.pl/ kazienko/mgrwytyczne.pdf [16.02.2017] http://www.pisanie-prac.info.pl/prace-magi-sterskie-jak-pisac.php [16.02.2017] 
Lesaw Cirko and Karin Pittner - 9783631776346 Downloaded from PubFactory at 03/06/2019 10:19:32AM

via free access 